\title{
Elevated Serum Estradiol Levels Have Different Effects on Pregnancy Outcomes Depending on Whether Cleavage-stage or Blastocyst-stage Embryo Transfer Is Performed During Artificial Frozen Embryo Transfer Cycles
}

\section{Qing Li}

Nanning Maternal and Child Health Care Hospital

\section{Liming Ruan}

Nanning Maternal and Child Health Care Hospital

\section{Lingling Zhu}

Maternal and Child Health Policy Research Center

\section{Zengyu Yang}

Maternal and Child Health Policy Research Center

\section{Maoling Zhu}

Nanning Maternal and Child Health Care Hospital

Yudi Luo ( $\nabla$ yudiluo_ylfy@163.com )

Yulin Maternal and Child health Care Hospital https://orcid.org/0000-0003-1943-5031

\section{Research}

Keywords: blastocyst, cleavage-stage embryo, estradiol, frozen embryo transfer, hormone replacement treatment, ongoing pregnancy/live birth rate

Posted Date: December 28th, 2020

DOI: https://doi.org/10.21203/rs.3.rs-134071/v1

License: (c) (1) This work is licensed under a Creative Commons Attribution 4.0 International License.

Read Full License 


\section{Abstract}

Objective: The aim of this study was to evaluate the association between serum estradiol $\left(E_{2}\right)$ and pregnancy outcomes of cleavage- or blastocyst-stage frozen embryo transfer (FET) cycles using hormone replacement therapy.

Methods: A total of 776 FET cycles (669 couples) performed from January 2016 to December 2019 were included in the present retrospective cohort study. The impact of progesterone-initiation-day serum $E_{2}$ levels on the ongoing pregnancy/live birth (OP/LB) rates was determined, and cleavage-stage embryo transfers and blastocyst-stage embryo transfers were analyzed separately.

Results: Regarding cleavage-stage embryo transfer cycles, serum $E_{2}$ levels on progesterone initiation day were significantly lower in the OP/LB group than in the non-OP/LB group (214.75 \pm 173.47 vs. $253.20 \pm$ $203.30 \mathrm{pg} / \mathrm{ml} ; P=0.023)$. In addition, there were downward trends in implantation, clinical pregnancy and $\mathrm{OP} / \mathrm{LB}$ rates with increasing $\mathrm{E}_{2}$ levels. However, in blastocyst-stage embryo transfer cycles, such trends were not observed, and there was no significant difference between the OP/LB group and the non-OP/LB group. Logistic regression analysis revealed that $\mathrm{E}_{2}$ levels on progesterone initiation day in cleavagestage embryo transfer cycles were independently associated with OP/LB (odds ratio $=1.000,95 \%$ confidence interval: 1.000-1.001, $P=0.008$ ). The areas under the receiver operating characteristic curve were 0.55 in cleavage-stage embryo transfer cycles and 0.53 in blastocyst-stage embryo transfer cycles.

Conclusions: The association of low OP/LB rates with elevated $E_{2}$ levels on the progesterone initiation day in cleavage-stage embryo transfer cycles suggests that $E_{2}$ levels should be monitored during artificial cleavage-stage embryo transfer cycles. However, it is not necessary to monitor serum $E_{2}$ levels when transferring blastocysts in artificial FET cycles.

\section{Introduction}

Endometrial preparation is a critical step in both the natural and artificial frozen embryo transfer (FET) cycle because the development of the endometrium must be synchronized with the embryo transfer for successful implantation [1]. Undoubtedly, estradiol $\left(\mathrm{E}_{2}\right)$ coordinately interacts with progesterone and plays an important role in endometrial development $[2,3]$. In fresh cycles, excess $E_{2}$ has been shown to be detrimental to endometrial development and to ultimately adversely affect conception $[4,5]$. Moreover, excess $E_{2}$ levels have been found to increase the incidence of abnormal pregnancy conditions, such as intrauterine growth restriction and abnormal implantation of the placenta [6, 7]. There is limited available information regarding the need for endocrine monitoring during hormone replacement therapy (HRT), although supplementation with steroid hormones is necessary for endometrial preparation. Fritz et al. demonstrated that elevated $\mathrm{E}_{2}$ levels in artificial FET cycles were related to a low ongoing pregnancy/live birth (OP/LB) rate [8], but some authors did not observe this association [9-11]. The discrepant findings may be due to the different patient populations recruited, differences in the protocols used for 
endometrial preparation, and differrences in the day in which steroid hormone levels were measured between studies. In addition to synchronization between endometrial maturation and transferred embryos, female age, the uterine condition, and embryonic factors are major variables affecting embryo implantation. To eliminate bias due to the effects of other variables on embryonic implantation, the present retrospective study analyzed frozen-thawed transfer cycles with two good-quality embryos transferred into the normal uterus of patients under 40 years of age with an endometrial thickness of at least $7 \mathrm{~mm}$ on the progesterone initiation day. Cleavage-stage embryo transfer cycles and blastocyststage embryo transfer cycles were analyzed separately in this study because the age of the transferred embryo is an important factor affecting the success rate of implantation. To our knowledge, to date, this is the largest study examining the association between $E_{2}$ levels on progesterone initiation day and OP/LB.

\section{Material And Methods}

\section{Patients}

All FET cycles performed from January 2016 to December 2019 were retrospectively analyzed in this study. A total of 776 cycles (669 couples) that met the following criteria were included: women under 40 years of age, transfer of two viable-quality embryos, and endometrial thickness $\geq 7 \mathrm{~mm}$ on the progesterone initiation day. Among day-3 embryos, those with 6 to 10 even and homogeneous blastomeres and less than $40 \%$ fragmentation were defined as viable embryos. Blastocysts were assessed on day 5 or day 6 after oocyte collection according to Gardner's criteria [12], and all blastocysts except for CC (poor inner cell mass and poor trophectoderm) were defined as viable embryos.

Cryopreservation and thawing of embryos were performed with the Cryotop (Kitazato Supply Co., Japan) protocol reported by Kuwayama et al (2005) [13]. Patients with hydrosalpinx or uterine abnormalities such as fibroids or congenital structural anomalies that may affect implantation were excluded. The present study was approved by the hospital ethical board (study number SZ20200319-1).

\section{Endometrial preparation and embryo transfer}

Endometrial preparation started on days 2 to 3 of the cycle with a step-up dose of oral $E_{2}$ valerate (Progynova, Bayer, France) with or without downregulation by gonadotropin-releasing hormone agonist. In general, $4 \mathrm{mg}$ per day of estradiol valerate was administered for the first 4 days, and then increased to $6 \mathrm{mg}$ per day for the second 4 days, followed by the measurement of endometrial thickness by vaginal ultrasound. If endometrial thickness reached $7 \mathrm{~mm}$, administrtion of the same dose of valerate was continued for 4 more days, and if not, the doses were increased to $8 \mathrm{mg}$ per day onwards. When the thickness reached $7 \mathrm{~mm}$ after 12-15 days of $E_{2}$ valerate administration, progesterone supplementation was administered by three routes based on patient preference: oral administration of $10 \mathrm{mg}$ twice a day (Dydrogesterone, Abbott, Netherlands), 90 mg vaginal administration daily (Crinone, MerckSerono, UK) or $40 \mathrm{mg}$ intramuscular injection daily (Progesterone Injection; Xianjupharmacy, China). All patients underwent transfers of 2 embryos under ultrasound guidance with day-3 embryos 4 days after 
progesterone administration or blastocysts 6 days after progesterone administration. Serum levels of $E_{2}$, progesterone and luteinizing hormone were measured on the progesterone initiation day using electrochemiluminescent immunoassay (Roche, Cobase, Switzerland). Human chorionic gonadotropin was examined to evaluate pregnancy on days 12-14 after embryo transfer. When pregnancy was achieved, the combination of $E_{2}$ valerate and progesterone supplementation continued until 10-12 weeks of gestation.

\section{Pregnancy outcomes}

The presence of a gestational sac and fetal heartbeat was considered a clinical pregnancy. The implantation rate was calculated as the number of gestational sacs by ultrasound observation divided by the number of transferred embryos. Live birth was defined as delivery of a viable infant after 24 weeks gestation. Miscarriage was diagnosed as spontaneous pregnancy loss.

\section{Statistical analysis}

Statistical analysis was conducted with SPSS software (version 17.0; SPSS Inc., Chicago, IL, USA). Continuous values are presented as the mean \pm SD. Categorical values are expressed as percentages (\%). Normality was assessed with the Shapiro-Wilk test. Student's t-test or Mann-Whitney U test were used to evaluate differences between continuous variables based on the distribution. Categorical values were analyzed with the chi-square test. Multivariate logistic regression analysis with stepwise backwards regression was performed to assess the correlations between the variables and the OP/LB rate. All the variables reaching a significance level of $10 \%$ with the chi-square test were enterred into the logistic regression. The area under the receiver operating characteristic $(\mathrm{ROC})$ curve was calculated to determine the predictive power of serum $\mathrm{E}_{2}$ level for OP/LB. A two-tailed $P$ value $<0.05$ was considered statistically significant.

\section{Results}

A total of 776 FET cycles (669 patients) were retrospectively reviewed from January 2016 to December 2019. The implantation rate, clinical pregnancy rate (CPR) and OP/BL rate were $27.4 \%, 43.3 \%$ and $33.5 \%$, respectively, for cleavage-stage embryo transfer cycles and $48.1 \%, 69.3 \%, 59.2 \%$, respectively, for blastocyst-stage embryo transfer cycles.

As shown in Table 1 for cleavage-stage embryo transfer cycles, there were no significant differences between $\mathrm{OP} / \mathrm{BL}$ and non-OP/BL regarding body mass index, type of infertility, basal serum folliclestimulating hormone levels on cycle days $2-3$, serum levels of progesterone and luteinizing hormone, or endometrial thickness on progesterone initiation day. However, the average female age, serum $\mathrm{E}_{2}$ level on progesterone initiation day, and duration of infertility were significantly lower in the OP/BL group than in the non-OP/BL group (31.9 vs. 33.4 years, $P<0.001 ; 214.75$ vs. $253.20 \mathrm{pg} / \mathrm{ml}, P<0.05 ; 4.34$ vs. 4.99 years, $P<0.05$; respectively). For blastocyst-stage embryo transfer cycles, no significant differences in any of the variables were observed between the OP/BL and non-OP/BL groups (Table 2). 
Table 1

Characteristics between ongoing pregnancy/live birth (OP/LB) and non-OP/LB for cleavage-stage embryo transfers.

\begin{tabular}{|c|c|c|c|}
\hline Parameters & $\begin{array}{l}\text { OP/LB group }(n \\
=180)\end{array}$ & $\begin{array}{l}\text { Non-OP/LB group }(n \\
=358)\end{array}$ & $\begin{array}{l}P \\
\text { value }\end{array}$ \\
\hline Female age (years) & $31.94 \pm 3.84$ & $33.39 \pm 3.82$ & $\dot{0} 001$ \\
\hline Body mass index & $22.11 \pm 2.77$ & $21.98 \pm 2.84$ & 0.628 \\
\hline Basal follicle-stimulating hormone (IU/L) & $6.03 \pm 2.35$ & $6.22 \pm 2.64$ & 0.432 \\
\hline Type of infertility & & & 0.848 \\
\hline Primary & $33.8 \%$ & $33.0 \%$ & \\
\hline Secondary & $66.2 \%$ & $67.0 \%$ & \\
\hline Duration of infertility (years) & $4.34 \pm 3.03$ & $4.99 \pm 3.71$ & 0.044 \\
\hline $\begin{array}{l}\text { Estradiol on the progesterone initiation day } \\
(\mathrm{pg} / \mathrm{mL})\end{array}$ & $214.75 \pm 173.47$ & $253.20 \pm 203.30$ & 0.023 \\
\hline $\begin{array}{l}\text { Progesterone on the progesterone initiation day } \\
(\mathrm{pg} / \mathrm{mL})\end{array}$ & $0.35 \pm 1.52$ & $0.27 \pm 0.74$ & 0.418 \\
\hline $\begin{array}{l}\text { Luteinizing hormone on the progesterone } \\
\text { initiation day }(\mathrm{IU} / \mathrm{L})\end{array}$ & $8.61 \pm 9.48$ & $7.25 \pm 8.03$ & 0.106 \\
\hline $\begin{array}{l}\text { Endometrial thickness on the progesterone } \\
\text { initiation day }(\mathrm{mm})\end{array}$ & $9.23 \pm 1.21$ & $9.11 \pm 1.22$ & 0.251 \\
\hline
\end{tabular}


Table 2

Characteristics between ongoing pregnancy/live birth (OP/LB) and non-OP/LB for blastocyst-stage embryo transfers.

\begin{tabular}{|c|c|c|c|}
\hline Parameters & $\begin{array}{l}\text { OP/LB group }(n \\
=141)\end{array}$ & $\begin{array}{l}\text { Non- OP/LB group } \\
(n=97)\end{array}$ & $\begin{array}{l}P \\
\text { value }\end{array}$ \\
\hline Female age (years) & $31.56 \pm 4.27$ & $31.84 \pm 4.25$ & 0.626 \\
\hline Body mass index & $21.99 \pm 3.05$ & $22.22 \pm 3.11$ & 0.573 \\
\hline Basal follicle-stimulating hormone (IU/L) & $5.47 \pm 1.81$ & $5.53 \pm 2.26$ & 0.810 \\
\hline Type of infertility & & & 0.534 \\
\hline Primary & $56.6 \%$ & $43.4 \%$ & \\
\hline Secondary & $60.6 \%$ & $39.4 \%$ & \\
\hline Duration of infertility (years) & $4.11 \pm 2.85$ & $3.85 \pm 2.39$ & 0.465 \\
\hline $\begin{array}{l}\text { Estradiol on the progesterone initiation day } \\
(\mathrm{pg} / \mathrm{mL})\end{array}$ & $201.66 \pm 182.14$ & $197.89 \pm 212.83$ & 0.884 \\
\hline $\begin{array}{l}\text { Progesterone on the progesterone initiation day } \\
(\mathrm{pg} / \mathrm{mL})\end{array}$ & $0.197 \pm 0.160$ & $0.208 \pm 0.168$ & 0.607 \\
\hline $\begin{array}{l}\text { Luteinizing hormone on the progesterone } \\
\text { initiation day (IU/L) }\end{array}$ & $7.67 \pm 8.19$ & $7.62 \pm 9.12$ & 0.966 \\
\hline $\begin{array}{l}\text { Endometrial thickness on the progesterone } \\
\text { initiation day }(\mathrm{mm})\end{array}$ & $8.95 \pm 1.29$ & $9.04 \pm 1.37$ & 0.630 \\
\hline
\end{tabular}

To further investigate serum $E_{2}$ levels on the effect of pregnancy outcomes, patients were classified into three percentile groups based on the serum $E_{2}$ level on progesterone initiation day: the 1st-10th percentile (group 1), the 11th-90th percentile (group 2) and the 91st-100th percentile (group 3). As shown in Fig. 1, we observed decreasing trends in the implantation rate $(P<0.001), \operatorname{CPR}(P=0.001)$ and $\mathrm{OP} / \mathrm{BL}$ rate $(P=0.024)$ with increasing $\mathrm{E}_{2}$ levels for cleavage-stage embryo transfer cycles. A significantly higher $O P / B L$ rate was achieved in cycles with $E_{2}$ levels in the $1 \mathrm{st}-10$ th percentile $(2.7-47.1 \mathrm{pg} / \mathrm{ml})$ than in cycles with $\mathrm{E}_{2}$ levels in the $91 \mathrm{st}-100$ th percentile $(508.4-951.0 \mathrm{pg} / \mathrm{ml})(43.4 \mathrm{vs} .18 .9 \%, P=0.006)$. However, such trends were not observed for the implantation rate, CPR or OP/BL rate in blastocyst-stage embryo transfer FET cycles (Fig. 2).

Multivariate logistic regression analysis with a stepwise backwards approach was performed to assess the correlations between the OP/BL rate and the variables for which the $P$ value was under $10 \%$, as shown in Table 1. These variables included female age, duration of infertility, and serum $E_{2}$ and luteinizing hormone levels on progesterone initiation day for cleavage-stage embryo transfer cycles. The results revealed that female age (odds ratio $(O R)=1.059,95 \%$ confidence interval $(95 \% \mathrm{Cl}): 1.003-1.118$, $P=0.039)$ and serum $\mathrm{E}_{2}$ level on progesterone initiation day $(\mathrm{OR}=1.000,95 \% \mathrm{Cl}: 1.000-1.001, P=0.008)$ 
were independently associated with the OP/BL rate for cleavage-stage embryo transfer cycles (Table 3). Logistic regression analysis for blastocyst-stage embryo transfer cycles was not performed because no $P$ value was under 0.10, as shown in Table 2.

Table 3

Valuables associated with ongoing pregnancy/live birth analyzed with multivariate logistic regression for day-3 embryo transfers.

\begin{tabular}{|llll|}
\hline Valuables & Odds ratio & 95\% confidence interval & $P$-value \\
\hline Female age & 1.059 & $1.003-1.118$ & 0.039 \\
\hline Estradiol level on the progesterone initiation day & 1.000 & $1.000-1.001$ & 0.008 \\
\hline Constant & 0.234 & & 0.120 \\
\hline
\end{tabular}

In addition, $\mathrm{ROC}$ analysis was performed to determined whether the serum $\mathrm{E}_{2}$ level on progesterone initiation day could predict OP/BL. The areas under the ROC curve were 0.55 (95\% Cl: $0.50-0.60)$ for cleavage-stage embryo transfer cycles (Fig. 3 ) and 0.53 (95\% Cl: $0.46-0.61)$ for blastocyst-stage embryo transfer cycles (Fig. 4), implying that the serum $E_{2}$ level on progesterone initiation day is a poor predictive variable for $\mathrm{OP} / \mathrm{BL}$ in FET cycles.

\section{Discussion}

We demonstrated that the serum $\mathrm{E}_{2}$ level on the progesterone initiation day was significantly higher in the OP/LB group than in the non-OP/LB group during artificial FET cycles with cleavage-stage embryo transfer. In addition, elevated $\mathrm{E}_{2}$ levels in the 91st-100th percentile had detrimental effects on embryo implantation, clinical pregnancy and OP/LB compared to $E_{2}$ levels in the 1st-10th percentile. However, such effects of $E_{2}$ levels on embryo implantation, clinical pregnancy and OP/LB were not observed in frozen-thawed blastocyst-stage embryo transfer cycles. This is the first study to find that serum $E_{2}$ levels on progesterone initiation day have different effects on pregnancy outcomes between cleavage-stage embryo transfers and blastocyst-stage embryo transfers during artificial FET cycles.

Although estrogen levels in normal natural cycles reach $300-400 \mathrm{pg} / \mathrm{ml}$ before ovulation, a study on donor cycles revealed that the $E_{2}$ requirement for embryo implantation is low ( $\left.<100 \mathrm{pg} / \mathrm{ml}\right)$ [14]. Our study supports this conclusion, showing appropriately $40 \%$ of conceptions occurring in patients with $E_{2}$ levels $<100 \mathrm{pg} / \mathrm{ml}$ either in cleavage-stage embryo transfers (95/233) or in blastocyst-stage embryo transfer cycles (65/165). On the other hand, successful conception in fresh embryo transfer cycles implies that embryo implantation can occur in an environment with the supraphysiological levels of $E_{2}$ caused by ovarian stimulation. This can be explained by the fact that serum $E_{2}$ levels were not associated with pregnancy outcomes in blastocyst transfer cycles in the present study. Similarly, in a study by Özdemir et al., $\mathrm{E}_{2}$ levels did not have significant effects on the pregnancy and miscarriage rate in 
autologous day 5 embryo transfer cycles using HRT [10]. Our data showed that pregnancies could occur in a wide range of serum $E_{2}$ levels from low levels of $10 \mathrm{pg} / \mathrm{ml}$ to a supraphysiological level of > $1000 \mathrm{pg} / \mathrm{mL}$.

Interestingly, regarding cleavage-stage embryo transfer, we found that elevated $E_{2}$ levels in the 91 st-100th percentile on progesterone initiation day had detrimental effects on CPR and OP/LB rates in HRT cycles compared to the low $\mathrm{E}_{2}$ levels in the 1st-10th percentile. Fritz et al. had a similar finding that OP/LB decreased six-fold in FET cycles with the highest $10 \% \mathrm{E}_{2}$ concentrations compared to those cycles with the lowest $10 \% \mathrm{E}_{2}$ concentrations [8]. The detrimental effects of an elevated $\mathrm{E}_{2}$ level on pregnancy have been reported in fresh in vitro fertilization cycles $[4,5,15]$. Experiments with a mouse model revealed that high levels of estrogen rapidly close the receptivity window for embryo implantation by altering endometrial gene expression, which causes the uterus to become unreceptive for implantation [16, 17]. As a consequence of this shortened receptivity window caused by a high $\mathrm{E}_{2}$ level, the receptivity window may be missed for a late-growing blastocyst developed from a day-3 embryo, resulting in a low chance of implantation; however, a blastocyst transfer can result in immediate implantation within the receptivity window.

In contrast to our findings regarding high $\mathrm{E}_{2}$ levels in cleavage-stage FET cycles, some authors have not found an adverse effect of high $E_{2}$ levels on pregnancy outcomes. Niu et al. reported that $E_{2}$ concentrations did not appear to be associated with the pregnancy rate in autologous day-3 FET cycles [9]. They grouped $E_{2}$ concentrations on progesterone initiation day into the $0-25$ th, 25th -75 th, and 75 th100th percentile groups, and the average $E_{2}$ concentration in the highest group $(299 \pm 48.9 \mathrm{pg} / \mathrm{ml})$ was not in the range of the highest 10th percentile of our study $(508.4-951.0 \mathrm{pg} / \mathrm{ml})$. When we regrouped $\mathrm{E}_{2}$ levels according to their study, no differences in pregnancy or implantation between groups were observed. Classifying $E_{2}$ levels into five groups, Remohi et al. did not find elevated $E_{2}$ levels on the oocyte donation day to be associated with the implantation or pregnancy rate [14]. This may be due to the high quality of embryos derived from donor oocytes that can be implanted within a narrow receptive window in conditions of elevated $E_{2}$ levels, which was similar to our findings for blastocyst-stage embryo transfer cycles.

In contrast to our finding of a significant difference in $E_{2}$ levels between the OP/LB group and the nonOP/LB group for cleavage-stage embryo transfer cycles, this difference in $E_{2}$ levels was not found by several authors $[8,11,18]$. This discrepancy may be have two main causes. First, these authors used transdermal and intramuscular $E_{2}$ for $E_{2}$ supplementation, whereas we used oral estrogen. Oral estrogen is metabolized and transformed into estrone in the intestine and liver, which has a lower binding affinity for estrogen receptors than $E_{2}$ [19]. Second, the study by Fritz et al. pooled day-3 and day- 5 embryos for analysis [8], whereas we analyzed data for cleavage-stage embryo transfer and blastocyst-stage embryo transfer separately. 
Multivariable logistic regression was conducted to analyze $E_{2}$ levels on progesterone initiation day in cleavage-stage embryo transfer cycles. We found that the $E_{2}$ level was an independent variable affecting OP/LB after adjustments for female age. However, ROC curve analysis revealed that the $E_{2}$ level on progesterone initiation day had no predictive power for pregnancy outcomes in either cleavage-stage or blastocyst-stage embryo transfer cycles. Although we acknowledge that the retrospective nature is a limitation in this study, recruitment of women under 40 years old with two good embryos being transferred into a normal endometrium eliminated the bias caused by other confounding variables. Another limitation involving the small sizes of the highest and lowest $E_{2}$ levels indicates the need for larger sample sizes in prospective studies in the future.

\section{Conclusions}

Our data indicate that excess serum $\mathrm{E}_{2}$ levels on the progesterone initiation day have detrimental effects on embryo implantation and pregnancy rates when cleavage-stage frozen embryos are transferred into the endometrium prepared by oral administration of $E_{2}$ but have no adverse effect on frozen blastocyststage embryo transfer. Therefore, it is necessary to monitor $E_{2}$ levels in cleavage-stage embryo transfer cycles but not necessary in blastocyst-stage embryo transfer cycles. However, serum $E_{2}$ levels on progesterone initiation day could not predict success in either cleavage- or blastocyst-stage HRT frozen embryo transfer cycles.

\section{Abbreviations}

$\mathrm{E}_{2}$ : estradiol; FET: frozen embryo transfer cycle; HRT: hormone replacement therapy; CPR: clinical pregnancy rate; OP/LB: ongoing pregnancy/live birth; OR: odds ratio; $95 \% \mathrm{Cl}$ : $95 \%$ confidence interval; ROC: receiver operating characteristic

\section{Declarations}

\section{Acknowledgments}

The authors would like to thank the staff at the reproductive medicine center in Yulin Maternal and Child Health Care Hospital for their support.

\section{Funding}

Not applicable.

\section{Availability of data and materials}

The datasets used and analyzed during the current study are available from the corresponding author on reasonable request. 


\section{Authors' Contributions}

Design of the study: QL, LR, YL and MZ. Acquisition of data: QL, ZY, YL. Analyzed and interpreted the data: QL, LR, ZY, LZ, YL and MZ. Manuscript writing and revising: QL, YL and MZ. All authors read and approved the final manuscript.

\section{Ethical approval}

All procedures involving human participants were conducted in accordance with those of the 1964 Helsinki declaration and with the recommendations of the institutional review boards of Yulin Maternal and Child Health Care Hospital. This study was approved by the Yulin Maternal and Child Health Care Hospital (NO. SZ20200319-1). Patient consent was not required because this was a retrospective study.

\section{Consent for publication}

Not applicable.

\section{Competing interests}

The authors declare that there are no conflicts of interest.

\section{References}

1. Paria B, Reese J, Das SK, Dey SK. Deciphering the cross-talk of implantation: advances and challenges. Science. 2002;296(5576):2185-8.

2. Albrecht ED, Aberdeen GW, Pepe GJ. The role of estrogen in the maintenance of primate pregnancy. Am J Obstet Gynecol. 2000;182(2):432-8.

3. Rashid NA, Lalitkumar S, Lalitkumar PG, Gemzell-Danielsson K. Endometrial receptivity and human embryo implantation. Am J Reprod Immunol. 2011;66:23-30.

4. Forman R, Fries N, Testart J. Evidence for an adverse effect of elevated serum estradiol concentrations on embryo implantation. Fertil Steril. 1988;27(3):476-.

5. Simón C, Cano F, Valbuena D, Remohí J, Pellicer A. Clinical evidence for a detrimental effect on uterine receptivity of high serum oestradiol concentrations in high and normal responder patients. Hum Reprod. 1995;10(9):2432-7.

6. Farhi J, Haroush AB, Andrawus N, Pinkas H, Sapir O, Fisch B, et al. High serum oestradiol concentrations in IVF cycles increase the risk of pregnancy complications related to abnormal placentation. Reprod BioMed Online. 2010;21(3):331-7.

7. Imudia AN, Awonuga AO, Doyle JO, Kaimal AJ, Wright DL, Toth TL, et al. Peak serum estradiol level during controlled ovarian hyperstimulation is associated with increased risk of small for gestational age and preeclampsia in singleton pregnancies after in vitro fertilization. Fertil Steril. 2012;97(6):1374-9. 
8. Fritz R, Jindal S, Feil H, Buyuk E. Elevated serum estradiol levels in artificial autologous frozen embryo transfer cycles negatively impact ongoing pregnancy and live birth rates. J Assist Reprod Genet. 2017;34(12):1633-8.

9. Niu Z, Feng Y, Sun Y, Zhang A, Zhang H. Estrogen level monitoring in artificial frozen-thawed embryo transfer cycles using step-up regime without pituitary suppression: is it necessary? J Exp Clin Assist Reprod. 2008;5(1).

10. Özdemir AZ, Karli P, Gülümser Ç. Does high estrogen level negatively affect pregnancy success in frozen embryo transfer? Arch Med Sci. 2019;16(1):https://doi.org/10.5114/aoms.2020.92466.

11. Bocca S, Real EB, Lynch S, Stadtmauer L, Beydoun H, Mayer J, et al. Impact of serum estradiol levels on the implantation rate of cleavage stage cryopreserved-thawed embryos transferred in programmed cycles with exogenous hormonal replacement. J Assist Reprod Genet. 2015;32(3):395400.

12. Gardner DK, Lane M, Stevens J, Schlenker T, Schoolcraft WB. Blastocyst score affects implantation and pregnancy outcome: towards a single blastocyst transfer. Fertil Steril. 2000;73(6):1155-8.

13. Kuwayama M, Vajta G, leda S, Kato O. Comparison of open and closed methods for vitrification of human embryos and the elimination of potential contamination. Reprod BioMed Online. 2005;11(5):608-14.

14. Remohi J, Ardiles G, Garcia-Velasco J, Gaitán P, Simón C, Pellicer A. Endometrial thickness and serum oestradiol concentrations as predictors of outcome in oocyte donation. Hum Reprod. 1997;12(10):2271-6.

15. Arslan M, Bocca S, Arslan EO, Duran HE, Stadtmauer L, Oehninger S. Cumulative exposure to high estradiol levels during the follicular phase of IVF cycles negatively affects implantation. J Assist Reprod Genet. 2007;24(4):111-7.

16. Ma W-g, Song H, Das SK, Paria BC, Dey SK. Estrogen is a critical determinant that specifies the duration of the window of uterine receptivity for implantation. Proc Natl Acad Sci USA. 2003;100(5):2963-8.

17. Simon C, Domínguez F, Valbuena D, Pellicer A. The role of estrogen in uterine receptivity and blastocyst implantation. Trends Endocrinol Metab. 2003;14(5):197-9.

18. Banz C, Katalinic A, Alhasani S, Seelig AS, Weiss JM, Diedrich K, et al. Preparation of cycles for cryopreservation transfers using estradiol patches and Crinone $8 \%$ vaginal gel is effective and does not need any monitoring. Eur J Obstet Gynecol Reprod Biol. 2002;103(1):43-7.

19. Paulson RJ. Hormonal induction of endometrial receptivity. Fertil Steril. 2011;96(3):530-5.

\section{Figures}




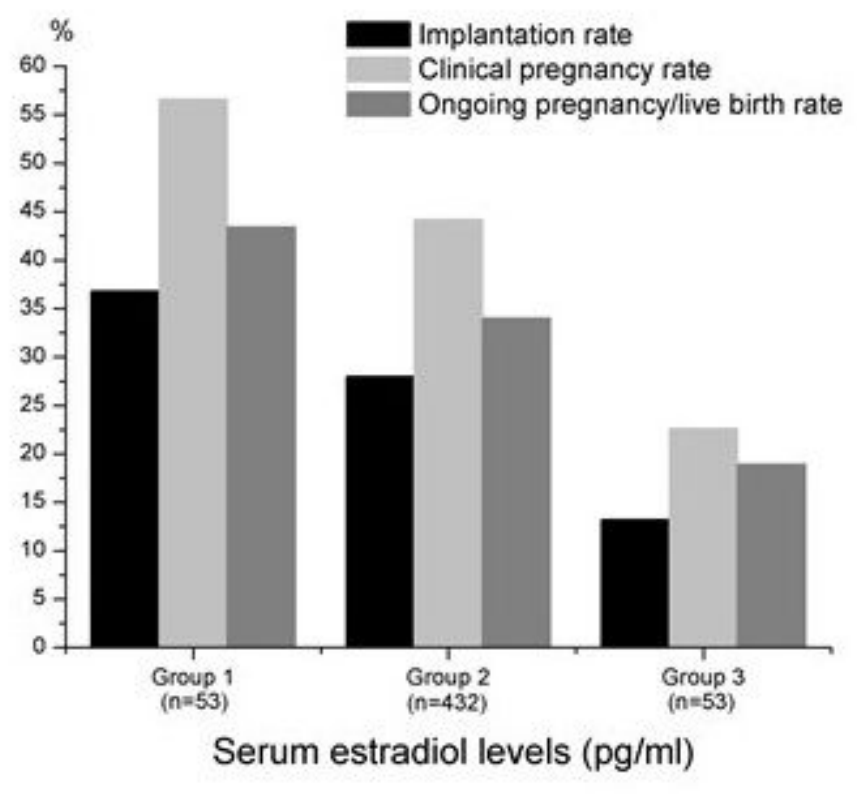

Figure 1

Pregnancy outcomes for cleavage-stage embryo transfer cycles. Serum estradiol levels are classified into three percentile groups: group 1, the 1st-10th percentile; group 2, the 11th-90th percentile; and group 3 , the 91 st -100 th percentile. $n$ represents cycle number in each group. P-values are $<0.001,0.001,0.024$ for implantation rate, clinical pregancy rate and ongoing pregnancy/live birth rate. 


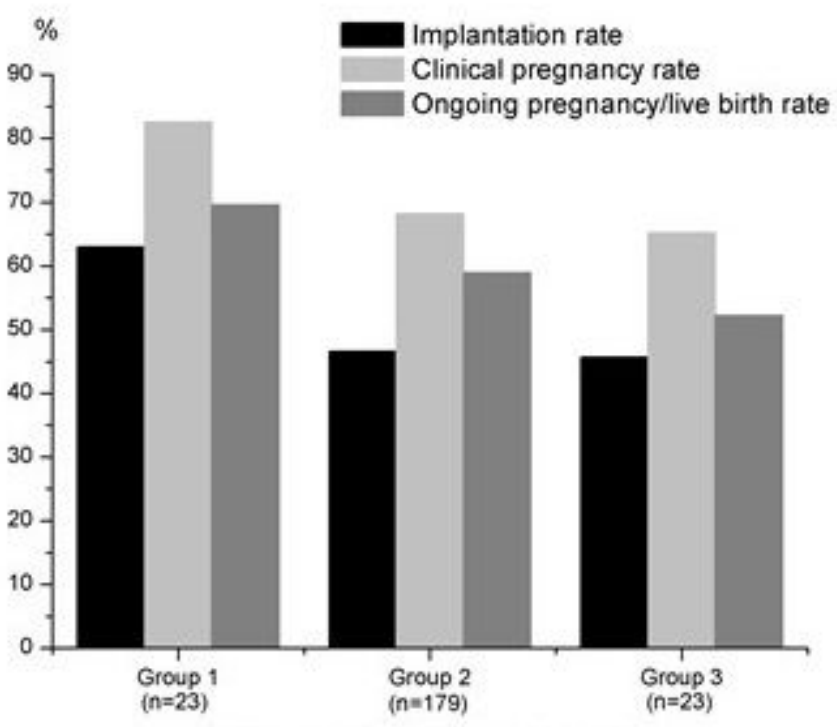

Serum estradiol levels $(\mathrm{pg} / \mathrm{ml})$

\section{Figure 2}

Pregnancy outcomes for blastocyst-stage embryo transfer cycles. Serum estradiol levels are classified into three percentile groups: group 1, the 1st-10th percentile; group 2, the 11th-90th percentile; and group 3 , the 91 st-100th percentile. $n$ represents cycle number in each group. P-values are 0.102, 0.333, 0.472 for implantation rate, clinical pregancy rate and ongoing pregnancy/live birth rate. 


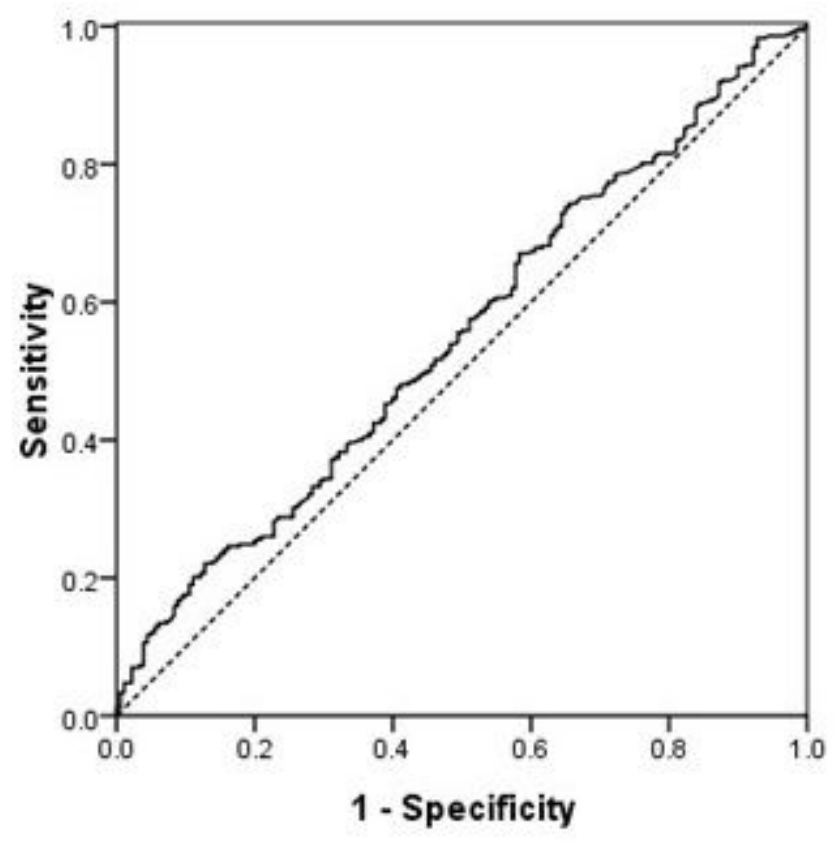

Figure 3

Receiver operator characteristic curve for prediction of ongoing pregnancy/live birth for cleavage-stage embryo transfers. The area under the curve is 0.55 . 


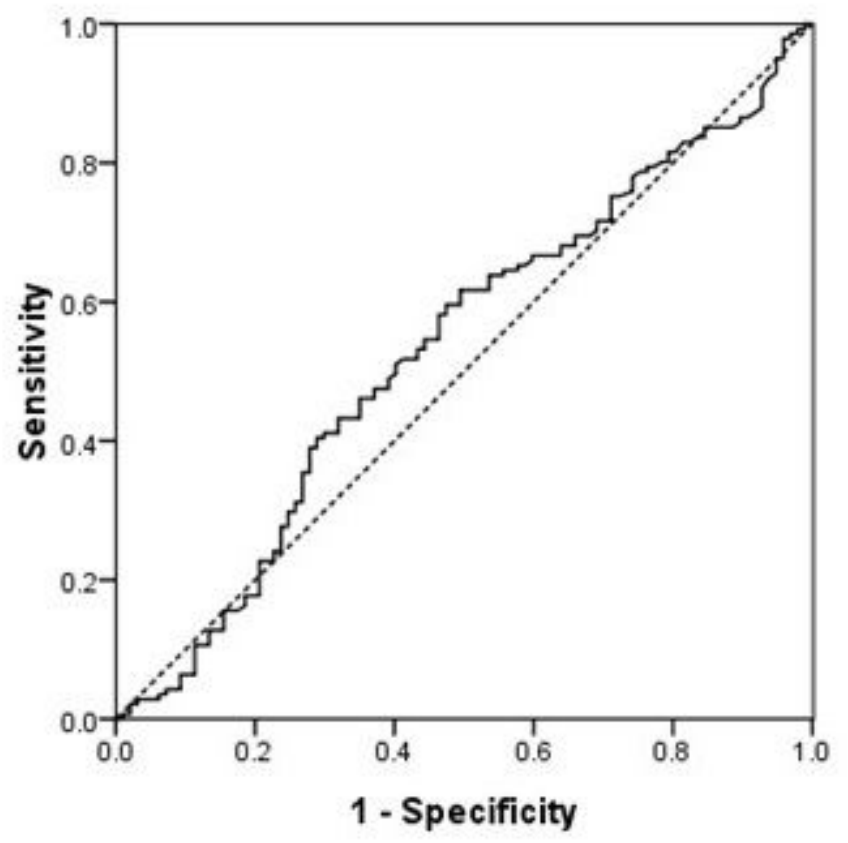

Figure 4

Receiver operator characteristic curve for prediction of ongoing pregnancy/live birth for blastocyst-stage embryo transfers. The area under the curve is 0.53 . 